\title{
How delivery mode and feeding can shape the bacterial community in the infant gut
}

\author{
Se Jin Song BS, Maria Gloria Dominguez-Bello PhD, Rob Knight PhD
}

See related research article by Azad and colleagues on page 385 and at www.cmaj.ca/lookup/doi/10.1503/cmaj.121189

I n Canada, $27 \%$ of newborns are delivered surgically, compared with $18 \%$ in 1990 . This rising trend is global: according to recent surveys, the proportion born by cesarean delivery has surged to more than $30 \%$ in the United States, higher in Mexico and several European countries, and to nearly $50 \%$ in China and Brazil. ${ }^{1}$ Many cesarean deliveries are performed electively, despite high costs and risks to the mother. When combined with low rates of exclusive breastfeeding, ${ }^{2}$ these practices are of growing concern as we continue to discover the ways in which mode of delivery and infant diet can affect both short- and long-term health outcomes.

Epidemiologic evidence has shown a correlation between cesarean delivery and obesity, asthma, celiac disease and type 1 diabetes. ${ }^{3}$ Conversely, breastfeeding has been associated with a decreased risk of obesity, diabetes and diarrheal diseases. ${ }^{4}$ Although the exact mechanisms remain to be elucidated, these correlations appear to stem from differences in gut microbial communities. ${ }^{5}$ In a related CMAJ article, Azad and colleagues have characterized the gut microbiota in 24 healthy term infants. ${ }^{6}$ They note that, despite the high variability in microbiota profiles between the infants, the relative abundance of several bacterial groups differed significantly by mode of delivery and by infant diet. Specifically, they, like other investigators, found that formulafed infants (regardless of their breastfeeding status) had increased richness of species, with overrepresentation of Clostridium difficile. Azad and colleagues also found that infants born by elective cesarean delivery had particularly low bacterial richness and diversity.

The findings of Azad and colleagues have important implications. Early interactions with beneficial bacteria train the immune system of babies and are thus critical for healthy immunologic and metabolic programming. ${ }^{7}$ All mammals are naturally inoculated as they pass through the birth canal, which guarantees exposure to the mother's vaginal bacteria first, before other environmental sources. They then drink maternal milk exclusively for a substantial proportion of their development, which further enriches their microbial community. Breast milk, rich in prebiotics, selects for persistence of beneficial bacteria and limits colonization of harmful ones. The strong selective pressure of breast milk is demonstrated by the lower diversity of microbes in the gut of breastfed infants than in the gut of formula-fed infants, as noted by Azad and colleagues. ${ }^{6}$

These conserved mammalian traits are likely adaptive. Also, this natural system is highly synchronized at the microbial level as the mother's body prepares to provide a specific inoculum. In one study, the microbiota in breast milk differed between women who delivered vaginally and those who underwent elective cesarean delivery. ${ }^{8}$ In another study, Lactobacillus johnsonii, a species of bacteria normally found in the gut, was more abundant in the vaginal microbial communities of pregnant women than in those of nonpregnant women. ${ }^{9}$ Because of the known enzymatic activity of these bacteria, it has been suggested that they, once ingested by the neonate during vaginal delivery, may then play an important role in shaping the bacterial community colonizing the infant gut.

Studies of microbiota in mothers and infants have consistently shown that deviation from traditional methods of delivery and feeding can alter the microbiota in both mother and infant. Although we do not yet fully understand how changes in microbial diversity or composition affect microbiome function, certain types of dis-

\section{KEY POINTS}

- The infant gut is particularly susceptible to alterations of the resident microbiota.

- Changes in the developing microbiota have been associated with breastfeeding, cesarean delivery and antibiotic use.

- These changes in the developing microbiome could explain susceptibilities to a variety of conditions later in life.

- Women and medical professionals should be aware of these issues when planning delivery and neonatal care. 
ruption during development may have lifelong effects on the baby's microbiome and thus the individual. Antibiotic use - an increasingly prevalent practice in Western societies - may also negatively affect the long-term health of infants by reducing the number of beneficial bacteria and impeding recolonization to a normal microbial community. ${ }^{10}$ Children born by cesarean delivery or fed with formula may be at increased risk of a variety of conditions later in life; ${ }^{3,4}$ both processes alter the gut microbiota in healthy infants, which could be the mechanism for the increased risk. These issues are of direct relevance to pregnant women and health practitioners and should be considered when choices such as elective cesarean delivery and other interventions are discussed.

Our microbiomes are complex and dynamic ecosystems, affected by interactions between our genetic predispositions, our behaviour and the environment. Consequently, what constitutes an "ideal" microbiome is not yet clear: it is not simply a matter of diversity, stability or even function. Studies have shown that our microbiota can be modified - perhaps irreversibly in some instances - by medical, dietary and hygienic practices. Infants may be particularly sensitive to the effects of such practices because of the vulnerability of their developing microbiomes and immune systems. ${ }^{5}$

A logical next step will be to continue investigating larger populations in well-designed prospective longitudinal studies that follow the same individuals from birth through childhood. The results will help us gain a better understanding of the many forms that a "normal" microbiome can take, the many ways in which it can be altered, and whether the changes result in positive or negative health outcomes. Deeper sequencing, including whole-genome sequencing of relevant strains and expression profiling, in conjunction with additional experimental research including animal models, will help to clarify the functional roles of our often underappreciated microbial communities.

\section{References}

1. Organisation for Economic Co-operation and Development. Caesarean sections. In: Health at a glance 2011: OECD indicators. Paris (France): OECD Publishing; 2011. Available: www.oecd.org/health/healthataglance (accessed 2013 Jan. 31).

2. World Health Organization. WHO global data bank on breastfeeding. Geneva (Switzerland): The Organization; 2011. Available: https://apps.who.int/nut/db_bfd.htm (accessed 2013 Jan. 28).

3. Hyde MJ, Modi N. The long-term effects of birth by caesarean section: the case for a randomised controlled trial. Early Hum Dev 2012;88:943-9.

4. Stuebe A. The risks of not breastfeeding for mothers and infants. Rev Obstet Gynecol 2009;2:222-31.

5. Madan JC, Farzan SF, Hibberd PL, et al. Normal neonatal microbiome variation in relation to environmental factors, infection and allergy. Curr Opin Pediatr 2012;24:753-9.

6. Azad MB, Konya T, Maughan H, et al.; the CHILD Study Investigators. Gut microbiota of healthy Canadian infants: profiles by mode of delivery and infant diet at 4 months. CMAJ 2013; 185:385-94

7. Maynard CL, Elson CO, Hatton RD, et al. Reciprocal interactions of the intestinal microbiota and immune system. Nature 2012;489:231-41

8. Cabrera-Rubio R, Collado MC, Laitinen K, et al. The human milk microbiome changes over lactation and is shaped by maternal weight and mode of delivery. Am J Clin Nutr 2012;96:544-51.

9. Aagaard K, Riehle K, Ma J, et al. A metagenomic approach to characterization of the vaginal microbiome signature in pregnancy. PLOS ONE 2012;7:e36466.

10. Fouhy F, Guinane CM, Hussey S, et al. High-throughput sequencing reveals the incomplete, short-term recovery of infant gut microbiota following parenteral antibiotic treatment with ampicillin and gentamicin. Antimicrob Agents Chemother 2012; 56:5811-20.

Affiliations: Se Jin Song is with the Department of Ecology and Evolutionary Biology, University of Colorado, Boulder, Colo. Maria Gloria Dominguez-Bello is with the Department of Biology, University of Puerto Rico, Rio Piedras, Puerto Rico, and the Division of Translational Medicine, New York University School of Medicine, New York, NY. Rob Knight is with the Departments of Chemistry and Biochemistry and of Computer Science, University of Colorado, Boulder, Colo., and is a Howard Hughes Medical Institute Early Career Scientist.

Contributors: The authors contributed equally to the writing and revising of the manuscript and approved the final version submitted for publication. 